3

4

5

6

7

\title{
Myostatin inhibitory region of fish (Paralichthys olivaceus) myostatin-1 propeptide
}

Sang Beum Lee ${ }^{1,2}$, Jeong Hwan Kim ${ }^{1}$, Deuk-Hee Jin ${ }^{1}$, Hyung-Joo $\mathrm{Jin}^{1 *}$ and Yong Soo $\mathrm{Kim}^{2 *}$,

${ }^{1}$ Department of Marine Molecular Biotechnology, Gangneung-Wonju National University, 120 Gangneugn Dachangno, Gangneung, 210-702, Korea

${ }^{2}$ Department of Human Nutrition, Food and Animal Sciences, University of Hawaii, 1955 East-West Rd., Honolulu, HI 96822, USA

\section{*Corresponding authors}

Yong Soo Kim: ykim@hawaii.edu (Tel. 808-956-8335; Fax: 808-9564883)

Hyung-Joo Jin: hj-jin@gwnu.ac.kr (Tel. +82-33-640-2349; Fax. +82-33-640-2849)

Current address of S.B. Lee: Wide River Institute of Immunology, Seoul National University College of Medicine, 101 Dabyeonbat-gil, Hwachon-myeon, Hongcheon-gun, Gangwon-do 250-812, South Korea. 


\section{Abstract}

Myostatin (MSTN) is a potent negative regulator of skeletal muscle growth, and its activity is suppressed by MSTN propeptide (MSTNpro), the N-terminal part of MSTN precursor cleaved during post-translational MSTN processing. The current study examined which region of flatfish (Paralichthys olivaceus) MSTN-1 propeptide (MSTN1pro) is critical for MSTN inhibition. Six different truncated forms of MSTN1pro containing N-terminal maltose binding protein (MBP) as a fusion partner were expressed in E. coli, and partially purified by an affinity chromatography for MSTN-inhibitory activity examination. Peptides covering different regions of flatfish MSTN1pro were also synthesized for MSTN-inhibitory activity examination. A MBP-fused MSTN1pro region consisting of residues 45-100 had the same MSTN-inhibitory potency as the full sequence flatfish MSTN1pro (residues 23-265), indicating that the region of flatfish MSTN1pro consisting of residues 45-100 is sufficient to maintain the full MSTN-inhibitory capacity. A MBP-fused MSTN1pro region consisting of residues 45-80 (Pro45-80) also showed MSTN-inhibitory activity with a lower potency, and the Pro45-80 demonstrated its MSTN binding capacity in a pull-down assay, indicating that the MSTN-inhibitory capacity of Pro45-80 is due to its binding to MSTN. Flatfish MSTN1pro synthetic peptides covering residues 45-65, 45-70, and 45-80 demonstrated MSTN-inhibitory activities, but not the synthetic peptide covering residues 45-54, indicating that residues 45-65 of flatfish MSTN1pro is essential for MSTN inhibition. In conclusion, current study show that like the mammalian MSTNpro, the MSTN-inhibitory region of flatfish MSTN1pro resides near its N-terminus, and imply that smaller sizes of MSTNpro can be effectively used in various applications designed for MSTN inhibition.

Key words: Myostatin, myostatin propeptide, inhibitory region, truncated protein, Paralichthys olivaceus, fish myostatin propeptide 


\section{Introduction}

Myostatin (MSTN), a member of the TGF- $\beta$ superfamily, is a strong negative regulator of skeletal muscle growth and development (Lee, 2004; McPherron et al., 1997; Rodgers and Garikipati, 2008). Thus, there has been much interest in suppressing MSTN activity as a strategy to enhance skeletal muscle growth in meat-producing animals and to treat muscle wasting conditions in humans, and studies have shown that MSTN inhibition enhances skeletal muscle growth in lab animals (Joulia-Ekaza and Cabello, 2007; Lee, 2004; Rodgers and Garikipati, 2008).

Like many other TGF- $\beta$ family member proteins (Kingsley, 1994; Massague, 1990), MSTN is translated as a precursor protein (preproMSTN) composed of a signal sequence, an N-terminal propeptide domain and a C-terminal mature (active) domain (McPherron et al., 1997; Wolfman et al., 2003). After removal of the signal peptide, the proMSTN forms a disulfide-linked homodimer and is proteolytically processed at a conserved RXRR site by the furin family of proprotein convertases to generate propeptide and mature MSTN (Lee and McPherron, 2001; Thies et al., 2001). After cleavage, the disulfide-linked homodimer of mature MSTN is complexed with propeptide (MSTNpro), inhibiting MSTN activity by remaining in a latent/inactive state (Hill et al., 2002; Thies et al., 2001; Zimmers et al., 2002). Mammals and chicken possess single mstn gene, but fish possess multiple mstn genes as a result of at least two separate gene duplication events. The first duplication that occurred during early fish radiation produced two distinct mstn clades (mstn1 and 2), and another duplication occurred in each clade within salmonids, resulting in four mstn genes in salmonids (Garikipati et al., 2006; Kerr et al., 2005; Maccatrozzo et al., 2001; Ostbye et al., 2007; Rodgers and Garikipati, 2008). Unlike mammals, fish express MSTN in multiples tissues, suggesting a diverse functional role of MSTN in fish. Studies have shown that MSTN acts also as a negative regulator of muscle growth in fish (Acosta et al., 2005; Amali et al., 
2004; Amali et al., 2008; Carpio et al., 2009; Medeiros et al., 2009; Xu et al., 2003), and fish MSTN activity is also suppressed by MSTNpro (Lee et al., 2010; Medeiros et al., 2009; Rebhan and Funkenstein, 2008; Xu et al., 2003).

Amino acid sequences of mature MSTN is highly conserved among species with about $88 \%$ homology between mammalian and most fish species, but MSTNpro is much less conserved among species with about 50\% homology between the mammalian and most fish species (Rodgers and Garikipati, 2008). It has been shown that a human MSTNpro region containing amino acid sequences between 42 and 115 was critical for MSTN inhibition (Jiang et al., 2004). Since a wide sequence variation exists between MSTNpro of mammalian and fish species, it was contended that the functional region of fish MSTN1pro for MSTN inhibition can be different from that of mammalian MSTNpro. Therefore, the objective of this study was to investigate the critical region of flatfish (Paralichthys olivaceus) MSTN1pro for MSTN inhibition by in vitro examination of MSTN-inhibitory capacities of various truncated forms of MSTN1pro. Results of this study shows that MBP-fused flatfish MSTN1pro consisting of residues 45-100 is sufficient to maintain the full MSTN-inhibitory activity, and the region consisting of residues 45-65 is essential for MSTN inhibition.

\section{Materials and methods}

\subsection{Construction of expression vectors}

Six truncated forms of flatfish MSTN1pro cDNA were produced by polymerase chain reaction (PCR) using the cDNA of full sequence flatfish MSTN1pro (Lee et al., 2012) as a template. The truncated flatfish MSTN1pros were named Pro23-265, Pro45-100, Pro4590, Pro45-80, Pro55-100, and Pro65-100 following their starting and ending positions in the sequence. Primer pairs for cDNA synthesis are listed in Table 1, and amino acid sequences of the truncated MSTN1pro proteins can be found in Fig. 1. The amplified, truncated 
MSTN1pro cDNAs were inserted into the pMALc5x expression vector, which adds maltose binding protein (MBP) as a fusion partner at the $\mathrm{N}$-terminal side of expression constructs (New England Biolab, MA, USA). E. coli strain of K12 TB1 (New England Biolab, MA, USA) was transformed with the expression constructs separately by the heat-shock method, and spread on Luria-Bertani (LB) (1.2\% tryptone, $0.6 \%$ yeast extract and $0.8 \% \mathrm{NaCl})$ agar plates containing $50 \mu \mathrm{g} / \mathrm{mL}$ ampicillin. Colonies were screened for correct insertion using colony PCR analysis, and selected colonies were subjected to DNA sequence analysis.

\subsection{Expression and affinity purification of MBP-fused flatfish MSTN1pro proteins}

Selected colonies were grown in $5 \mathrm{~mL}$ LB media with $50 \mu \mathrm{g} / \mathrm{mL}$ ampicillin at $37^{\circ} \mathrm{C}$ overnight, and the $5 \mathrm{~mL}$ cultures were used to inoculate $400 \mathrm{~mL}$ LB media containing 50 $\mu \mathrm{g} / \mathrm{mL}$ ampicillin. When the cell density reached to an $\mathrm{OD}_{600}$ of $0.4 \sim 0.5$, IPTG was added to the cultures to a final concentration of $0.3 \mathrm{mM}$, then the cultures were grown at $37^{\circ} \mathrm{C}$ for $2 \mathrm{hr}$. Cell pellets were collected, and soluble fractions of cell lysates were prepared, and MBPfused MSTN1pro proteins were affinity-purified from cell lysates using amylose resin as described previously (Lee et al., 2012). After SDS-PAGE analysis of the presence of MSTN1pro proteins in elution, fractions containing the target protein were pooled and dialyzed in $20 \mathrm{mM}$ Tris-HCl buffer ( $\mathrm{pH} 8.0$ ), then stored at $-20^{\circ} \mathrm{C}$. Protein concentration was determined by the Modified Lowry Protein Assay (Thermo Scientific, CA, USA), using BSA as a standard.

\subsection{Examination of the Binding of MBP-fused flatfish MSTN1pro proteins to MSTN}

A pull-down assay was performed to examine the ability of MSTN1pro proteins to bind to MSTN. One microgram of mouse MSTN (R\&D Systems, MN, USA) was added to $10 \mu \mathrm{g}$ of affinity-purified MSTN1pro proteins, then mixed separately for $2 \mathrm{hr}$ at room temperature with $10 \mu \mathrm{L}$ of amylose resin equilibrated with a column buffer (200 mM NaCl, 20 mM Tris, 1 mM EDTA, pH 7.4) in a centrifugal spin column, followed by centrifugation 
at 4,000 $\mathrm{g}$ for $1 \mathrm{~min}$. After removing the pass-through, the resin was washed with $1 \mathrm{~mL}$ of column buffer three times. The resin was then mixed with $20 \mu \mathrm{L}$ of reducing SDS-PAGE loading buffer. After heating the sample at $100^{\circ} \mathrm{C}$ for $5 \mathrm{~min}$, the loading buffer was carefully removed from the resin. The eluted proteins were analyzed by SDS-PAGE and Western blotting.

\subsection{BMP-1 digestion of flat fish MSTN1pro and N-terminal amino acids sequence analysis} Full sequence flatfish MSTN1pro (Pro23-265, $100 \mu \mathrm{g}$ in $35 \mu \mathrm{L}$ of HEPES buffer (25 $\mathrm{mM}, \mathrm{pH} 7.5$ ) containing $0.01 \%$ Brij-35 was mixed with $500 \mathrm{ng}$ of human BMP-1 (R\&D Systems, MN, USA) in $5 \mu \mathrm{L}$ of the same buffer, followed by incubation for $1 \mathrm{hr}$ at $37^{\circ} \mathrm{C}$. Digestion by BMP-1 was examined by SDS-PAGE analysis. After confirmation of the digestion, C-terminal fragment band was cut and sent to a commercial lab (Alphalyte, Palo Alto, CA, USA) for N-terminal (5 amino acids) sequence analysis by Edman degradation. 2.5. Synthesis of peptides covering various regions of flatfish MSTN1pro

Five peptides covering different regions of flatfish MSTN1pro (Pep45-54, Pep45-65, Pep45-70, Pep45-80, and Pep45-100) were synthesized commercially using the Fmoc solid peptide synthesis procedure (Peptron Co., Daejeon, Korea) to examine the MSTN-inhibitory activity of those peptides. Mass spectrometry analysis (HP1100 LC/MSD System, Agilent Technologies Korea, Seoul, Korea) showed that the actual masses of each peptides were almost identical (more than 99\%) to the expected masses of the peptides, confirming correct synthesis of the peptides. The synthesized peptides were more than 95\% pure in a HPLC analysis (Prominence HPLC System, Shimadzu Korea, Seoul, Korea).

\subsection{SDS-PAGE}

SDS-PAGE (12.5\% polyacrylamide gel) was performed according to the method of Laemmli (Laemmli, 1970). Samples were mixed with loading buffer in the presence of 1.5\% $\beta$-mercaptoethanol and boiled for 5 min prior to loading on the gel. Bands were stained with 
153 Coomassie Brilliant blue solution.

154

155

156

157

158

159

160

161

162

163

164

165

166

167

168

169

170

171

172

173

174

175

176

177

178

\subsection{Western blot analysis}

Eluted proteins from the pull-down assay were fractionated by 15\% SDS-PAGE, transferred to a PVDF membrane by electro-blotting. The membrane was blocked with Trisbuffered saline (20 mM Tris-HCl, $150 \mathrm{mM} \mathrm{NaCl}$ at $\mathrm{pH}$ 7.5) plus $0.01 \%$ Tween 20 (TTBS) for $2 \mathrm{hr}$ at room temperature. The primary antibody was monoclonal anti-MSTN antibody (1:1000, MAB788, R\&D Systems, MN, USA) for overnight at $4^{\circ} \mathrm{C}$. Membrane was washed (3 ×10 min) with TTBS and incubated with HRP-conjugated anti-mouse IgG (1:5000, \#7076, Cell Signaling, MA, USA) for $3 \mathrm{hr}$ at room temperature. The membrane was washed (3 x 10 min), and HRP activity was detected using a chemiluminescence detection kit (Ez-Western Lumi Femto Kit, Daeil Lab Service, Seoul, Korea), and visualized by exposing to X-ray film. 2.8. pGL3-(CAGA) $)_{12}$-luciferase reporter assay

The capacities of truncated, MBP-fused MSTN1pro proteins and MSTN1pro peptides to inhibit MSTN activity were measured using the pGL3-(CAGA) ${ }_{12}$-firefly luciferase reporter assay in HEK293 cells stably expressing (CAGA) 12-luciferase gene construct (Cash et al., 2012). Cells were seeded on a 96-well culture plate at 40,000 cells/well in DMEM with 10\% fetal calf serum, penicillin-streptomycin plus fungizone, and grown for $24 \mathrm{hr}$ at $37^{\circ} \mathrm{C}$ with $5 \% \mathrm{CO}_{2}$. After removing the medium, MSTN ( $\left.1 \mathrm{nM}\right)$ plus various concentrations of commercial mouse MSTNpro (R\&D Systems, MN, USA), flatfish MSTN1pro proteins or MSTN1pro peptides in DMEM without serum were added to each well, and incubated for 24 hr. After removing the medium, $60 \mu \mathrm{L}$ of Bright-Glo luminescence substrate (Promega, Madison, WI, USA) were added, and incubated for 3 min. Luminescence was measured using Veritas microplate luminometer (Turner Biosystems Inc., CA, USA). The \% inhibition of MSTN activity was calculated by the following formula: $\%$ inhibition = (luminescence at 1 nM MSTN - luminescence at each ligand concentration)*100/(luminescence at 1 nM MSTN 
- luminescence at $0 \mathrm{nM}$ MSTN). The MSTN-inhibitory activities were analyzed by regression analysis using Prism5 program (Graphpad, San Diego, CA, USA). To examine the differences in MSTN-inhibitory capacity of these proteins and peptides, $\mathrm{IC}_{50}$ (ligand concentration inhibiting 50\% of MSTN activity) values were estimated using a non-linear regression model defining dose response curve. The equation for the model was as follows: Y $=$ Bottom $+($ Top - Bottom $) /\left(1+10^{\wedge}\left(\mathrm{X}-\operatorname{LogIC}_{50}\right)\right)$, where $\mathrm{Y}$ is \% inhibition, Bottom is the lowest value of \% inhibition, Top is the highest value of \% inhibition, and $\mathrm{X}$ is Log ligand concentration. IC $_{50}$ values were analyzed by ANOVA (Analysis of Variance) using the same program.

\section{Results}

\subsection{Purification of truncated, MBP-fused flatfish MSTN1pro proteins}

Six truncated forms of MSTN1pro, including Pro23-265, Pro45-100, Pro45-90, Pro45-80, Pro55-100, and Pro65-100, were expressed as MBP-fusion proteins. As was previously demonstrated with the full sequence of flatfish MSTN1pro (Lee et al., 2012), the above MSTN1pro proteins were expressed in soluble forms in E. coli (data not shown). The molecular masses of affinity-purified Pro23-268, Pro45-100, Pro45-90, Pro45-80, Pro55-100, and Pro65-100 in SDS-PAGE were in agreement with the expected molecular masses of 69, 48, 47, 46, 47, and $46 \mathrm{kDa}$, respectively (Fig. 2A).

\subsection{Bioactivity of truncated flatfish MSTN1pro proteins and their binding to MSTN}

MSTN-inhibitory capacities of affinity-purified MSTN1pro proteins were examined using a pGL3-(CAGA) 12 -luciferase reporter assay system (Fig. 2B). Pro23-265, Pro45-100, Pro45-90, and Pro45-80 inhibited MSTN activity, but Pro55-100 and Pro65-100 had no MSTN-inhibitory activity, suggesting that residues 45-54 of flatfish MSTN1pro is

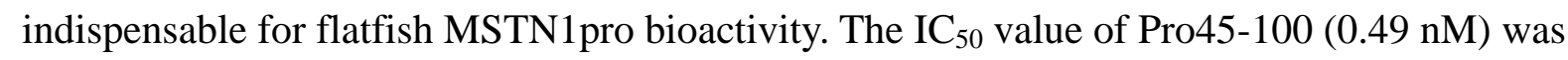
not different from that of Pro23-265 (0.57 nM), indicating that the region of MSTN1pro 
consisting of amino acid residues 45-100 is sufficient to maintain the full MSTN-inhibitory activity of flatfish MSTN1pro. The IC $_{50}$ values of Pro45-100 and Pro23-265 were significantly lower than that of a commercial mouse MSTNpro (1.02 nM), suggesting that flatfish MSTN1pro is more potent than mouse MSTNpro in inhibiting MSTN activity. The $\mathrm{IC}_{50}$ values of Pro45-90 (1.93 nM) and Pro45-80 (1.30 nM) were significantly higher than that of Pro45-100, indicating that the residues 81-100 is needed for full MSTN-inhibitory activity.

To examine whether the MSTN-inhibitory activity of flatfish MSTN1pro is through its binding to MSTN, MSTN-binding capacities of Pro45-80 and Pro55-100 were examined in a pull-down assay. While Pro45-80 showed its binding to MSTN, Pro55-100 did not show its binding to MSTN (Fig. 3A), suggesting that the binding of MSTN1pro to MSTN is responsible for its inhibition of MSTN activity. To compare the binding kinetic between MSTN and Pro45-80 to the kinetic of MSTN inhibition by Pro45-80, various concentrations of Pro45-80 were mixed with a fixed concentration of MSTN (3 nM), followed by a quantitation of MSTN binding to Pro45-80 by Western blotting (Fig. 3B and C). The MSTN binding saturation of Pro45-80 occurred at around $15 \mathrm{nM}$ with $3 \mathrm{nM}$ MSTN, indicating that about 5-fold of Pro45-80 was required for a full-binding of MSTN. When we examined the full inhibition of 1 nM MSTN activity by Pro45-80 in the pGL3-(CAGA) ${ }_{12}$-luciferase reporter assay, the full inhibition occurred at around $7 \mathrm{nM}$, indicating that about 7-fold of Pro45-80 was required for full inhibition of MSTN activity. Thus, the Pro45-80 concentration for full binding of MSTN is not much different from the concentration for full inhibition of MSTN, confirming that the binding of MSTN1pro to MSTN is responsible for inhibition of MSTN activity.

\subsection{Bioactivity of various synthetic peptides of flatfish MSTN1pro}

In order to further verify the differences in MSTN-inhibitory activities of the 
truncated forms of MBP-MSTN1pro proteins, we synthesized five MSTN1pro peptides covering different regions of flatfish MSTN1pro; Pep45-54, Pep45-65, Pep45-70, Pep45-80 and Pep80-100. MSTN-inhibitory capacities of those peptides were examined starting from a very high concentration (5 $\mu \mathrm{M})$. Pep45-54 and Pep80-100 did not show MSTN-inhibitory activity, but Pep45-65, Pep45-70 and Pep45-80 showed MSTN inhibitory activities with IC $_{50}$ values of 2.56, 0.52 and $0.34 \mu \mathrm{M}$, respectively (Fig. 4), suggesting that at least a region consisting of residues 45-65 is required to inhibit MSTN activity. The result on measurement of bioactivities of flatfish MSTN1pro proteins (Fig. 2) indicated that residues 45-54 was indispensable for MSTN-inhibition, but the current result with synthetic peptides suggests that the domain covering residues 45-54 alone is not sufficient for MSTN inhibition.

\subsection{BMP-1 digestion of MNP-fused flatfish MSTN1pro}

Pro23-265 was treated with BMP-1 to examine its cleavage by bone morphogenetic protein-1/tolloid (BMP-1/TLD) family of metalloproteinases (Fig. 5). The reaction resulted in cleavage of Pro23-265 into two fragments with MBP-fused N-terminal fragment at around 60 kDa (asterisk in Fig. 5) and C-terminal fragment at around 17 kDa (arrowhead in Fig.5) in SDS-PAGE analysis. The size of C-terminal fragment was smaller than an expected size (20 $\mathrm{kDa}$ ) deduced from the reaction of pig MBP-MSTNpro with BMP-1 in our previous study (Haq et al., 2013). To examine the cleavage site of flatfish MSTN1pro, five N-terminal amino acid residues of the C-terminal fragment $(17 \mathrm{kDa})$ were analyzed by Edman degradation. The analysis resulted in XEPEP, indicating that the cleavage site is between Ala-125 and Thr-126 in Fig. 1. The calculated molecular size of amino acid sequences 126-268 of flatfish MSTN1pro is $16.4 \mathrm{kDa}$, corresponding to $17 \mathrm{kDa}$ observed from SDS-PAGE analysis.

\section{Discussion}

In the current study, we observed that a region of MBP-fused flatfish MSTN1pro consisting 
of amino acid residues 45-100 is sufficient to maintain the full MSTN-inhibitory activity, as well as observing that MBP-fused residues 45-80 is effective in MSTN inhibition with a lower potency. With synthetic peptides, this study also revealed that residues 45-65 of flatfish MSTN1pro is at least required for MSTN inhibition. Similar to our current results, it was recently reported that a region of human MSTNpro consisting of residues 42-99 fully (98.1\%) suppressed MSTN activity in co-transfection experiments, as well as reporting that the essential region of human MSTNpro for MSTN inhibition was residues 42-70, which is homologous to residues 45-73 of flatfish MSTN1pro (Ohsawa et al., 2015). Furthermore, a synthetic mouse MSTNpro peptide covering residues 45-68 (homologous to residues 47-70 of flatfish MSTN1pro) was effective in suppressing MSTN activity (Takayama et al., 2015).

Our current results, thus, for the first time show that the location of essential MSTNinhibitory region of fish MSTN1pro is not much different from that of mammalian MSTNpro. In contrast to these results, a study reported that a fusion protein consisting of residues $42-$ 115 of human MSTNpro was essential for MSTN inhibition with no activity being observed with residues 42-98 (Jiang et al., 2004). The reason for this difference is not clear, but differences in experimental protocol, such as the use of different fusion partners during protein expression and different assay systems for MSTN inhibition, could be associated with the difference in results.

X-ray crystallographic structure of TGF- $\beta 1$ propeptide has been recently determined, and sequence alignment of various propeptides of TGF- $\beta$ family members has shown that these propeptides have a similar structural fold even though the amino acid sequences of the propeptide regions of TGF- $\beta$ family member proteins are variable in number and low in homology (Shi et al., 2011). Based on the sequence alignment, the region between amino acid sequences 40 to 100 of MSTNpro encompasses $\alpha 1$ helix, latency lasso, and $\alpha 2$ helix domains (Fig. 1). According to the three-dimensional structure of TGF- $\beta 1$ propeptide, the $\alpha 1$ helix was 
predicted to block the binding of TGF- $\beta 1$ to type I receptor, while the type II receptor binding is mostly blocked by the proline-rich latency lasso loop (Shi et al., 2011). The regions consisting of residues 45-80 of flatfish MSTN1pro and residues 42-70 of human MSTNpro encompass $\alpha 1$ helix, indicating that like TGF- $\beta 1$ propeptide, the $\alpha 1$ helix domain of MSTNpro is essential for inhibition of MSTN activity. In support, the region consisting of residues 42-70 of human MSTNpro interacted with MSTN as well as both type I and type II receptors of MSTN (ALK4 and ActRIIB) (Ohsawa et al., 2015). Given that the MSTNinhibitory potency of flatfish MSTN1pro and human MSTNpro were dramatically enhanced by the inclusion of the latency lasso and $\alpha 2$ helix domains, the latency lasso and $\alpha 2$ helix domains probably play a role in stabilizing the binding of MSTNpro to MSTN.

It is generally known that the principal mechanism for the activation of latent MSTN in vivo is the release of MSTN from the latent complex of MSTN and MSTNpro induced by the cleavage of MSTNpro between Arg-99 and Asp-100 (in human) by the bone morphogenetic protein-1/tolloid (BMP-1/TLD) family of metalloproteinases (Lee, 2008; Wolfman et al., 2003). Our current results, however, showed that in flatfish MSTN1pro, the cleavage by BMP-1 occurred between Ala-125 and Thr-126, suggesting that the location of BMP-1/TLD metalloproteinase cleavage site for fish MSTN1pro is different from its expected site obtained from sequence alignment with mammalian MSTNpro (Fig. 1). If the cleavage of MSTNpro by BMP-1/TLD is the sole mechanism for activation of MSTN from the latent complex, it is predicted that the mammalian MSTNpro consisting of residues 42-99 and flatfish MSTN1pro consisting of residues 45-100 have no MSTN-inhibitory activity. The results of Jiang et al. (2004) are in agreement with the prediction. However, in disagreement with the prediction, our current results as well as Ohsawa et al. (2015) show that the Nterminal region of MSTNpro that is separated from the cleavage by BMP-1/TLD proteases is able to bind to MSTN and suppress its activity in vitro, raising a question related to the 
proposed mechanism. Furthermore, intramuscular injections of synthetic peptides consisting of residues within the N-terminal region of cleavage site of MSTNpro enhanced muscle weight in mice (Ohsawa et al., 2015; Takayama et al., 2015), demonstrating that the Nterminal fragment of the BMP-1/TLD cleavage site is able to inhibit MSTN activity in vivo. These results together suggest that the mechanism for the activation of latent MSTN in vivo is potentially more complex than the simple cleavage of MSTNpro by BMP-1/TLD proteases, thus future studies are warranted to investigate the mechanisms involved in the activation of latent MSTN.

In summary, the current study shows that MBP-fused flatfish MSTN1pro consisting of residues 45-100 is sufficient to maintain the full MSTN-inhibitory activity, and the region consisting at least of residues 45-65 is essential for MSTN inhibition. These findings point to the potential of using smaller sizes of MSTNpro in various applications designed for MSTN inhibition. At the same time, current findings raise a question related to the activation of latent MSTN via simple cleavage of MSTNpro induced by BMP-1/TLD metalloproteinases, thus asking for future investigations.

\section{Acknowledgement}

This work was financially supported by USDA-NIFA grant 2010-34135-21229 and Gangneung-Wonju National University Research Fund (Sabbatical research fund, 2014).

\section{References}

Acosta, J., Carpio, Y., Borroto, I., Gonzalez, O., Estrada, M.P., 2005. Myostatin gene silenced by RNAi show a zebrafish giant phenotype. J Biotechnol 119, 324-331.

Amali, A.A., Lin, C.J., Chen, Y.H., Wang, W.L., Gong, H.Y., Lee, C.Y., Ko, Y.L., Lu, J.K., Her, G.M., Chen, T.T., Wu, J.L., 2004. Up-regulation of muscle-specific transcription 
factors during embryonic somitogenesis of zebrafish (Danio rerio) by knock-down of myostatin-1. Developmental dynamics : an official publication of the American Association of Anatomists 229, 847-856.

Amali, A.A., Lin, C.J., Chen, Y.H., Wang, W.L., Gong, H.Y., Rekha, R.D., Lu, J.K., Chen, T.T., Wu, J.L., 2008. Overexpression of Myostatin2 in zebrafish reduces the expression of dystrophin associated protein complex (DAPC) which leads to muscle dystrophy. Journal of biomedical science 15, 595-604.

Carpio, Y., Acosta, J., Morales, R., Santisteban, Y., Sanchez, A., Estrada, M.P., 2009. Regulation of body mass growth through activin type IIB receptor in teleost fish. Gen Comp Endocrinol 160, 158-167.

Cash, J.N., Angerman, E.B., Kattamuri, C., Nolan, K., Zhao, H., Sidis, Y., Keutmann, H.T., Thompson, T.B., 2012. Structure of myostatin.follistatin-like 3: N-terminal domains of follistatin-type molecules exhibit alternate modes of binding. The Journal of biological chemistry 287, 1043-1053.

Garikipati, D.K., Gahr, S.A., Rodgers, B.D., 2006. Identification, characterization, and quantitative expression analysis of rainbow trout myostatin-1a and myostatin-1b genes. J Endocrinol 190, 879-888.

Haq, W.Y., Kang, S.K., Lee, S.B., Kang, H.C., Choi, Y.J., Lee, C.N., Kim, Y.S., 2013. Highlevel soluble expression of bioactive porcine myostatin propeptide in E. coli. Appl Microbiol Biot 97, 8517-8527.

Hill, J.J., Davies, M.V., Pearson, A.A., Wang, J.H., Hewick, R.M., Wolfman, N.M., Qiu, Y., 2002. The myostatin propeptide and the follistatin-related gene are inhibitory binding proteins of myostatin in normal serum. The Journal of biological chemistry 277, 40735-40741.

Jiang, M.S., Liang, L.F., Wang, S., Ratovitski, T., Holmstrom, J., Barker, C., Stotish, R., 2004. 
Characterization and identification of the inhibitory domain of GDF-8 propeptide. Biochemical and biophysical research communications 315, 525-531.

Joulia-Ekaza, D., Cabello, G., 2007. The myostatin gene: physiology and pharmacological relevance. Current opinion in pharmacology 7, 310-315.

Kerr, T., Roalson, E.H., Rodgers, B.D., 2005. Phylogenetic analysis of the myostatin gene sub-family and the differential expression of a novel member in zebrafish. Evol Dev 7, 390-400.

Kingsley, D.M., 1994. The TGF-beta superfamily: new members, new receptors, and new genetic tests of function in different organisms. Genes \& development 8, 133-146.

Laemmli, U.K., 1970. Cleavage of structural proteins during the assembly of the head of bacteriophage T4. Nature 227, 680-685.

Lee, S.B., Kim, J.H., Jin, H.J., Shin, I.S., Kim, Y.S., 2012. High-yield production of bioactive flatfish (Paralichthys olivaceus) myostatin-1 prodomain in Escherichia coli. American Journal of Biochemistry and Biotechnology 8, 195-201.

Lee, S.B., Kim, Y.S., Oh, M.Y., Jeong, I.H., Seong, K.B., Jin, H.J., 2010. Improving rainbow trout (Oncorhynchus mykiss) growth by treatment with a fish (Paralichthys olivaceus) myostatin prodomain expressed in soluble forms in E. coli. Aquaculture 302, 270278.

Lee, S.J., 2004. Regulation of muscle mass by myostatin. Annual review of cell and developmental biology 20, 61-86.

Lee, S.J., 2008. Genetic analysis of the role of proteolysis in the activation of latent myostatin. Plos One 3, e1628.

Lee, S.J., McPherron, A.C., 2001. Regulation of myostatin activity and muscle growth. Proc Natl Acad Sci U S A 98, 9306-9311.

Maccatrozzo, L., Bargelloni, L., Cardazzo, B., Rizzo, G., Patarnello, T., 2001. A novel second 
myostatin gene is present in teleost fish. FEBS letters 509, 36-40.

Massague, J., 1990. The transforming growth factor-beta family. Annual review of cell biology 6, 597-641.

McPherron, A.C., Lawler, A.M., Lee, S.J., 1997. Regulation of skeletal muscle mass in mice by a new TGF-beta superfamily member. Nature 387, 83-90.

Medeiros, E.F., Phelps, M.P., Fuentes, F.D., Bradley, T.M., 2009. Overexpression of follistatin in trout stimulates increased muscling. American journal of physiology. Regulatory, integrative and comparative physiology 297, R235-242.

Ohsawa, Y., Takayama, K., Nishimatsu, S., Okada, T., Fujino, M., Fukai, Y., Murakami, T., Hagiwara, H., Itoh, F., Tsuchida, K., Hayashi, Y., Sunada, Y., 2015. The Inhibitory Core of the Myostatin Prodomain: Its Interaction with Both Type I and II Membrane Receptors, and Potential to Treat Muscle Atrophy. Plos One 10, e0133713.

Ostbye, T.K., Wetten, O.F., Tooming-Klunderud, A., Jakobsen, K.S., Yafe, A., Etzioni, S., Moen, T., Andersen, O., 2007. Myostatin (MSTN) gene duplications in Atlantic salmon (Salmo salar): evidence for different selective pressure on teleost MSTN-1 and -2. Gene 403, 159-169.

Rebhan, Y., Funkenstein, B., 2008. Inhibition of fish myostatin activity by recombinant fish follistatin and myostatin prodomain: Potential implications for enhancing muscle growth in farmed fish. Aquaculture 284, 231-238.

Rodgers, B.D., Garikipati, D.K., 2008. Clinical, agricultural, and evolutionary biology of myostatin: a comparative review. Endocrine reviews 29, 513-534.

Shi, M.L., Zhu, J.H., Wang, R., Chen, X., Mi, L.Z., Walz, T., Springer, T.A., 2011. Latent TGF-beta structure and activation. Nature 474, 343-U370.

Takayama, K., Noguchi, Y., Aoki, S., Takayama, S., Yoshida, M., Asari, T., Yakushiji, F., Nishimatsu, S., Ohsawa, Y., Itoh, F., Negishi, Y., Sunada, Y., Hayashi, Y., 2015. 
Identification of the minimum peptide from mouse myostatin prodomain for human myostatin inhibition. Journal of medicinal chemistry 58, 1544-1549.

Thies, R.S., Chen, T., Davies, M.V., Tomkinson, K.N., Pearson, A.A., Shakey, Q.A., Wolfman, N.M., 2001. GDF-8 propeptide binds to GDF-8 and antagonizes biological activity by inhibiting GDF-8 receptor binding. Growth Factors 18, 251-259.

Wolfman, N.M., McPherron, A.C., Pappano, W.N., Davies, M.V., Song, K., Tomkinson, K.N., Wright, J.F., Zhao, L., Sebald, S.M., Greenspan, D.S., Lee, S.J., 2003. Activation of latent myostatin by the BMP-1/tolloid family of metalloproteinases. Proc Natl Acad Sci U S A 100, 15842-15846.

Xu, C., Wu, G., Zohar, Y., Du, S.J., 2003. Analysis of myostatin gene structure, expression and function in zebrafish. J Exp Biol 206, 4067-4079.

Zimmers, T.A., Davies, M.V., Koniaris, L.G., Haynes, P., Esquela, A.F., Tomkinson, K.N., McPherron, A.C., Wolfman, N.M., Lee, S.J., 2002. Induction of cachexia in mice by systemically administered myostatin. Science 296, 1486-1488. 


\section{Figure Legends}

\section{Fig. 1. Amino acid sequences of flatfish myostatin propeptide aligned with those of} mouse and human. Underline, italic bold characters $(\mathrm{N})$, and italic bold (residues 125 for flatfish, 99 for human, and 100 for mouse) indicates signal sequence, N-Glycosylation site (N-X-S/T), and putative proteolytic site by BMP-1/TLD proteases, respectively. The shaded amino acid residues in flatfish sequence indicate the same sequence identity with the mammalian species. The domain names, including $\alpha 1$, latency lasso, $\alpha 2$, fastener, and $\beta 1$, are based on a publication by Shi et al. (2011).

Fig. 2. SDS-PAGE of affinity purified, MBP-fused, truncated forms of flatfish MSTN1 propeptide and inhibition of MSTN activities by those proteins. A) Amylose resin affinity-purified Pro23-265, Pro45-100, Pro45-90, Pro45-80, Pro55-100, and Pro65-100 were subjected to $12.5 \%$ SDS-PAGE, and proteins were visualized with Coomassie blue staining. B) HEK293 cells stably expressing (CAGA) 12 -luciferase gene construct were used. Various concentrations of MBP-MSTN1pro proteins in combination with $1 \mathrm{nM}$ MSTN were added to the HEK293 cells, followed by incubation for $24 \mathrm{~h}$. Medium was removed, and luminescence substrate was added, followed by luminescence measurement. The error bars represent SEM $(\mathrm{n}=3)$. The $\mathrm{IC}_{50}$ value means not sharing the same superscript are different at $\mathrm{P}<0.05$.

Fig. 3. SDS-PAGE analysis of the binding of Pro45-80 and Pro55-100 to MSTN in a pulldown assay. A) Amylose resin was mixed with either $10 \mu \mathrm{g}$ of Pro45-80 plus $1 \mu \mathrm{g}$ of MSTN (lane 1) or $10 \mu \mathrm{g}$ of Pro55-100 plus $1 \mu \mathrm{g}$ of MSTN (lane 2). After intensive washing, proteins bound to the amylose resin were eluted with reducing SDS-PAGE loading buffer, and eluted proteins were subjected to SDS-PAGE. Asterisk (*) indicates MSTN monomer. B) Amylose 
resin was mixed with $3 \mathrm{nM}$ of MSTN plus various concentrations of Pro45-80 in separate tubes. After intensive washing, proteins bound to the resin were eluted, and elutions were subjected to Western blot analysis by blotting against anti-MSTN antibody. C) The band intensity was quantified by densitometry, and the intensity was expressed as a ratio to MSTN control (3 nM).

\section{Fig. 4. Inhibition of MSTN activity by synthetic flatfish MSTN1pro peptides of various} regions. HEK293 cells stably expressing (CAGA) ${ }_{12}$-luciferase gene construct were used. Various concentrations of synthetic flatfish MSTN1pro peptides in combination with $1 \mathrm{nM}$ MSTN were added to the HEK293 cells, followed by incubation for 24 h. Medium was removed, and luminescence substrate was added, followed by luminescence measurement. The error bars represent SEM $(n=3)$. The $\mathrm{IC}_{50}$ value means not sharing the same superscript are different at $\mathrm{P}<0.05$.

\section{Fig. 5. BMP-1 digestion of flat fish MSTN1pro.}

Full sequence flatfish MSTN1pro (Pro23-265, $100 \mu \mathrm{g}$ ) was reacted with $500 \mathrm{ng}$ of human BMP-1, followed by incubation for $1 \mathrm{hr}$ at $37^{\circ} \mathrm{C}$. Digestion by BMP-1 was examined by SDS-PAGE analysis. C-terminal fragment band was cut for N-terminal five amino acids sequence analysis by Edman degradation, and the analysis resulted in XEPEP. Asterisk and arrowhead indicate N-terminal fragment and C-terminal fragment, respectively. 
Table 1. Primers used for pMALc5x expression vector construction of truncated flatfish myostatin-1 propeptides (MSTN1pro)

\section{Truncated}

473 MSTN1pro

474 (a.a. residue)

475

ccggaattcgagcaaactgcgaatgaagg

ccggaattctcatcctgactcgctttgggccgt

477

Pro45-100

ccggaattctgcgacgtccggcagcagata

ccggaattcttatcacagcacgtcgtactggtcgag

Pro45-90

ccggaattctgcgacgtccggcagcagata

ccggaattctcattagaagttgctgcagcggcggtg

Pro45-80

ccggaattctgcgacgtccggcagcagata

ccggaattctcattaggagctgcttcacaatgtctc

Pro55-100

ccggaattcgcgactaaacgcgatcatat

ccggaattcttatcacagcacgtcgtactggtcgag

Pro65-100

ccggaattcgagcaaactgcgaatgaagg

ccggaattcttatcacagcacgtcgtactggtcgag

482

483

Underline indicates EcoRI site used in the construction of expression vectors. 
Fig. 1.

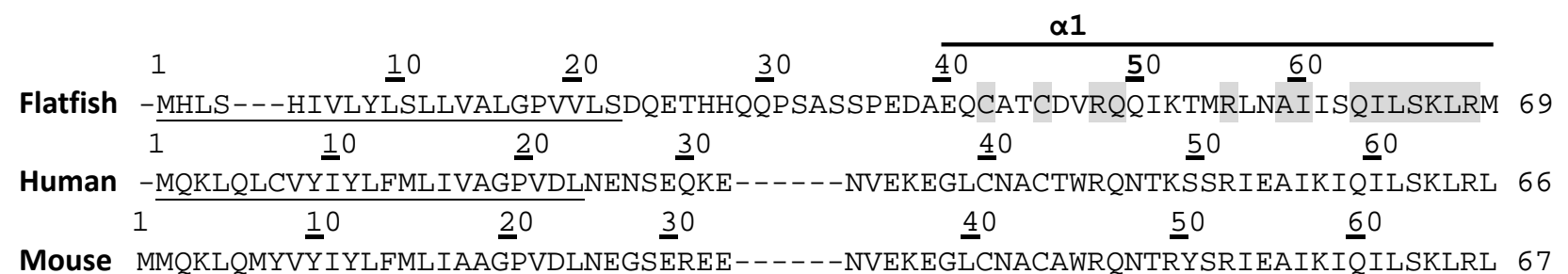

130

$11 \overline{10} \frac{1}{120}$

PIVQVDAEPK 139

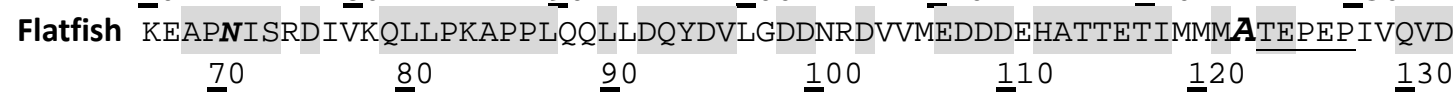

Human ETAP $N I S K D V I R Q \bar{L} L P K A P P L R E \bar{I} I D Q Y D V Q R D \overline{D S S D G S L E D D D Y H A T T E T I I T M P T E S D F L M Q}$ VDGKPK 136

$\begin{array}{lllllll}\underline{7} \odot & \underline{8} \Theta & \underline{9} \odot & 100 & 110 & \underline{1} 20 & \underline{1} 00\end{array}$

Mouse ETAPNISKDAIRQLLPRAPPLRELIDQYDVQRDDSSDGSLEDDDYHATTETIITMPTESDFLMQADGKPK 137
140
150
160
170
180
190
$\underline{2} 00$

Flatfish CCFFSFTQKFQASRIVRAQLWVYLRPADEATTVFLQISRLM-PVTDGSRHIRIRSLKIDVNAGLSSWQSID 209 $\begin{array}{lllllll}140 & 150 & 160 & 170 & 180 & 190 & \underline{2} 00\end{array}$

Human CCFFKFSSKIQYNKVVKAQLWIYLRPVETPTTVFVILRLIKPMKDGTRYTGIRSLKLDMNPGTGIWQSID 207
140
150
170
180
190 200

Mouse CCFFKFSSKIQYNKVVKAQLWIYLRPVKTPTTVFVQILRLIKPMKDGTRYTGIRSLKLDMSPGTGIWQSID 208

$\underline{210} \quad \underline{2} 20 \quad \underline{2} 30 \quad \underline{2} 40 \quad \underline{2} 50 \quad \underline{2} 60$

Flatfish VKQVLTVWLRQPETNWGIEINAFDSRGNDLAVTSTEPGEEGLQPFMEVKITDGPKRVRR 268 $\underline{210} \quad \underline{2} 20 \quad \underline{2} 30 \quad \underline{2} 40 \quad \underline{2} 50 \quad \underline{2} 60$

Human VKTVLQNWLKQPESNLGIEIKALDENGHDLAVTFPGPGEDGLNPFLEVKVTDTPKRSRR 266

$\begin{array}{llllll}210 & \underline{2} 20 & \underline{2} 30 & \underline{2} 40 & \underline{2} 50 & \underline{2} 6 \Theta\end{array}$

Mouse VKTVLQNWLKQPESNLGIEIKALDENGHDLAVTFPGPGEDGLNPFLEVKVTDTPKRSRR 267 
Fig. 2

A)

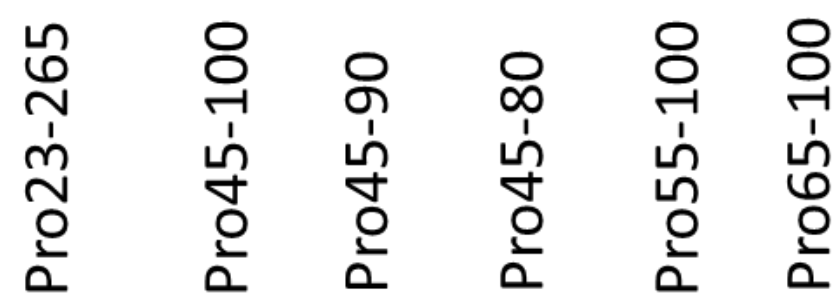

kDa

75

50

37

B)

$\mathrm{IC}_{50}$ for $1 \mathrm{nM}$ MSTN inhibition, $\mathrm{nM}$

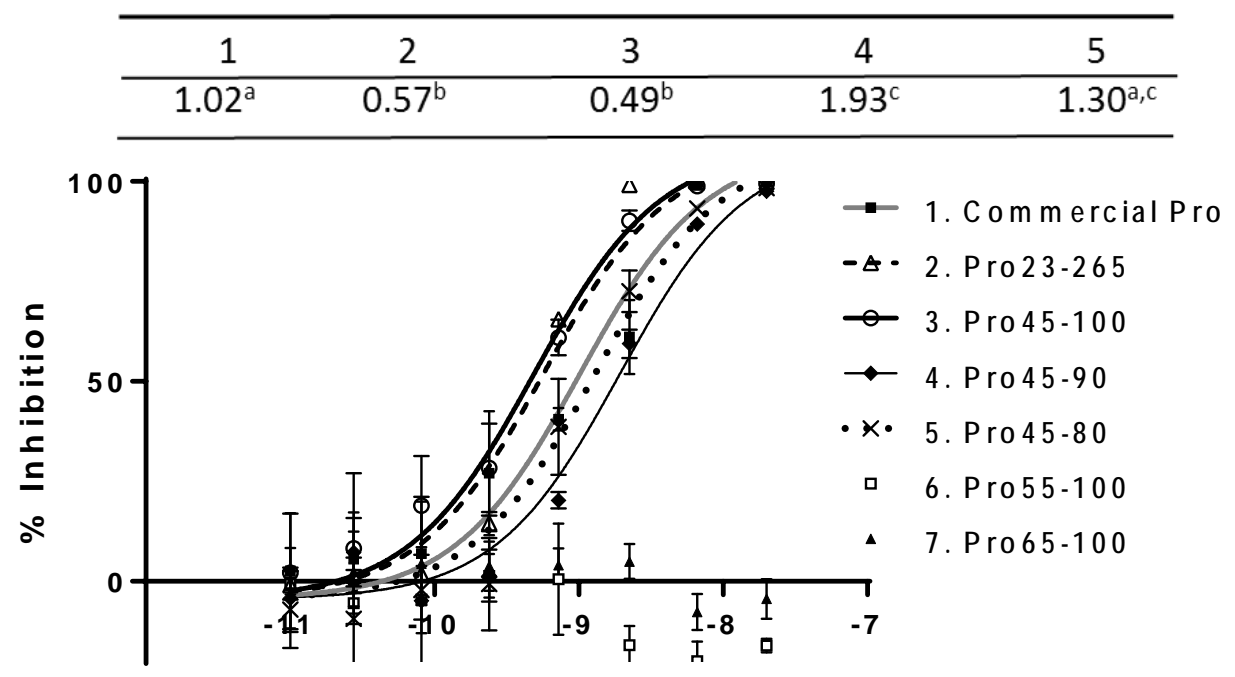

Ligand concentration ( $\log M)$ 
539

540

541

542

A)

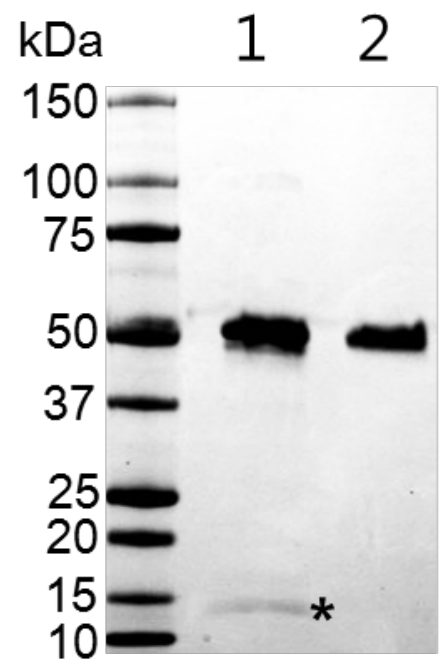

B) Pro45-80 concentration, nM MSTN

\begin{tabular}{lllllll}
\hline 0.6 & 3 & 6 & 15 & 30 & only \\
& & - & & & &
\end{tabular}

C)

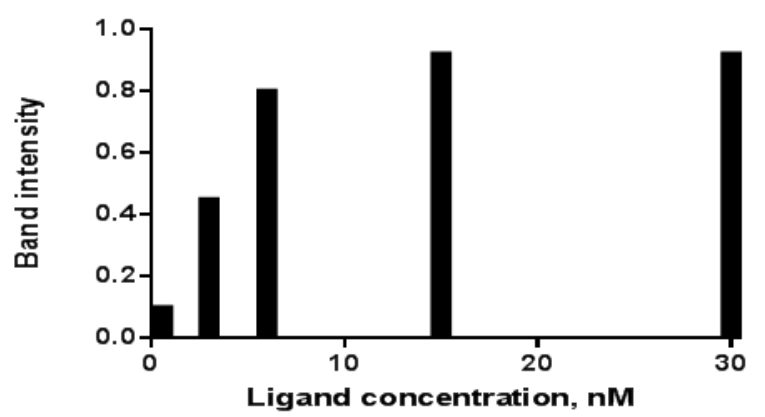

543

544

545

546

547

548

549

550

551

552

553

554

555

556

557

558

559

560

561 
562 Fig. 4.

563

564

$\mathrm{IC}_{50}$ for $1 \mathrm{nM}$ MSTN inhibition

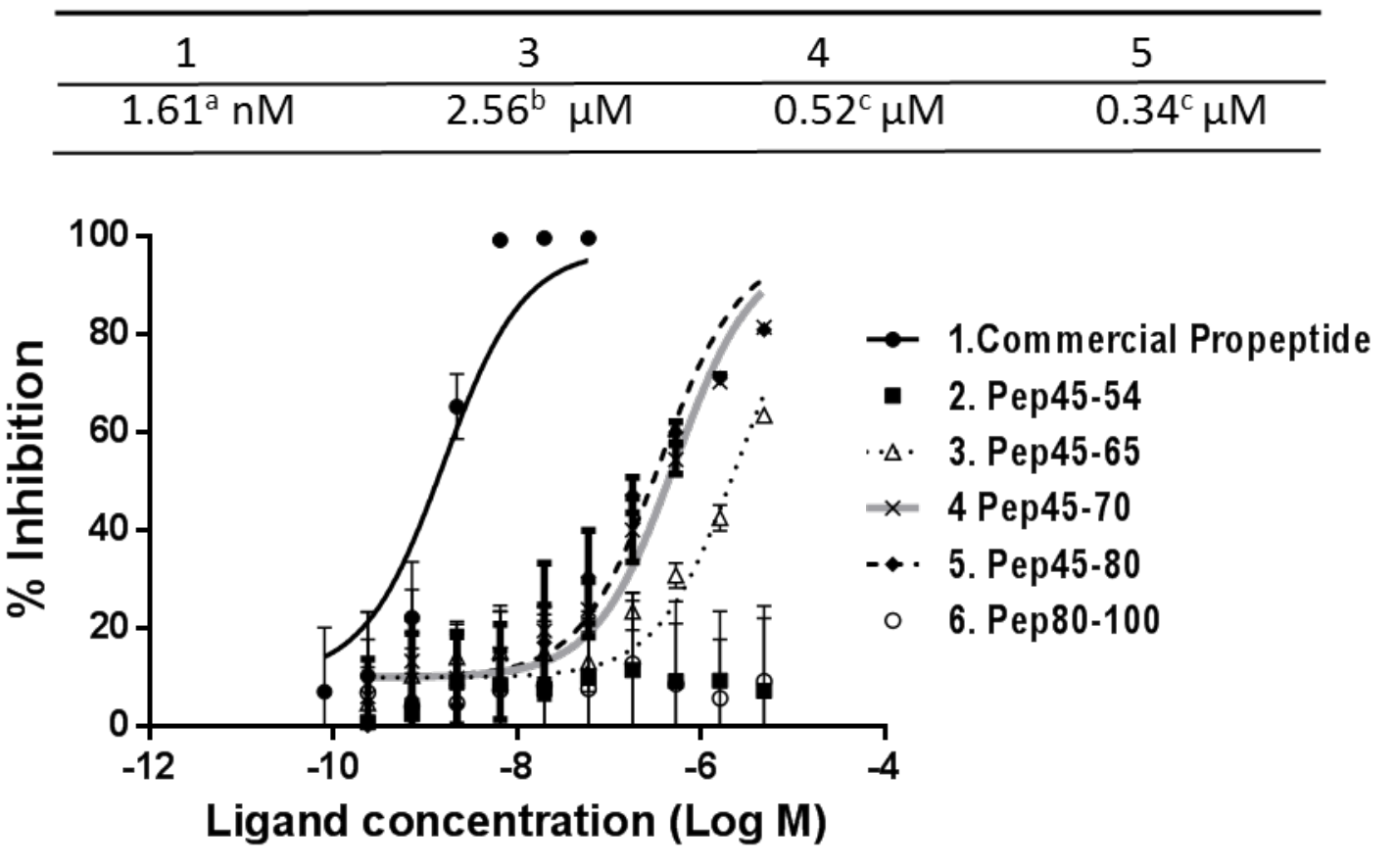

565

566

567

568

569

570

571

572

573

574

575 
576

577

578

579

580

581

Fig. 5.

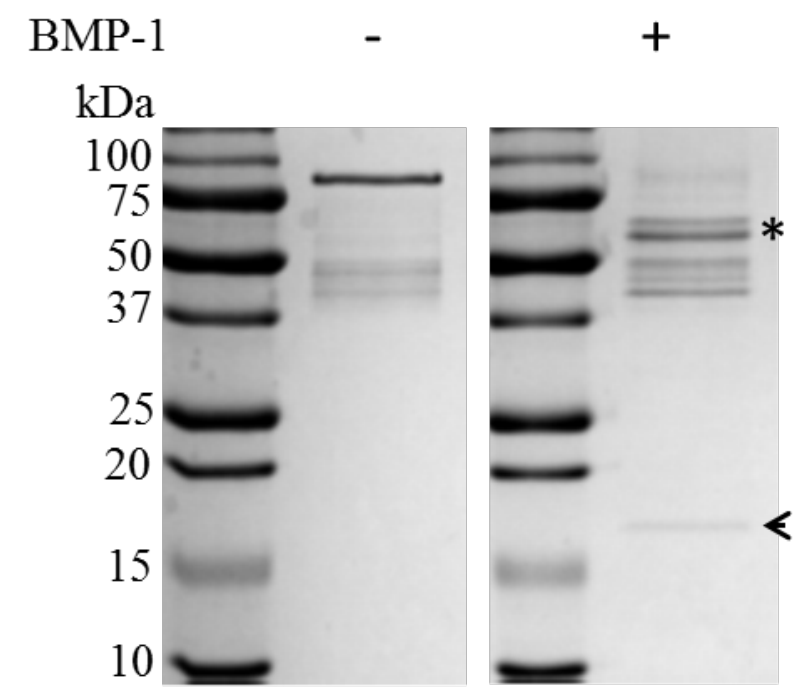

582

583

584

585

586

587

588

589

590

591 